\title{
President's Speech to the 2013 International Congress of Chinese Mathematicians
}

\author{
by Shing-Tung Yau \\ Department of Mathematics, Harvard University
}

Today is a festival celebrating the achievements of Chinese mathematicians. Fifteen years have passed by between the first congress in Beijing up until today in Taipei. Professor Lo Yang has a thorough understanding of the many hardships throughout these fifteen years, and yet, in spite of the difficulties, both academic and blood ties between scholars from Mainland China, Taiwan, Hong Kong, and overseas have fueled enthusiastic participation.

Through the five congresses held so far, scholars have not only received academic benefits, but they have also made many new friends. Chinese mathematicians have become a strong force in the worldwide mathematical community. Based on both the list of winners, as well as the content of the speeches, we can proudly say that our congress has truly reached the world-wide first-class level. There are, of course, still some less studied mathematical disciplines which require much more work, but our young scholars have certainly made outstanding achievements in them.

For example, in probability theory, Horng-Tzer Yau solved the Dyson conjecture. In number theory, we live in an era in which we no longer hesitate. We are no longer staying in the era of Luogeng Hua, Yuan Wang, Chengdong Pan, and Jingrun Chen. With the help of numerous overseas scholars such as John Coates, Shouwu Zhang, Ching-Li Chai, Jing Yu, Ju-Kang Yu, as well as many other Chinese and non-Chinese scholars, our young scholars have obtained important results over and over again in this important and classical field. This is definitely worthy of encouragement. Indeed, recently Yitang Zhang and Ye Tian have made enormous breakthroughs in classical problems in number theory. Their achievement dated back to the instruction and guidance of their tutors in Beijing: Professor Chengbiao Pan and Professor Chunlai Zhao.

Important achievements by Chinese mathematicians can also be found in algebraic geometry, algebra, and group representation theory, in which Chinese scholars such as Jun Li, Bong Lian, Chin-Lung Wang, Zhiwei Yun, Xuhua He, and Nanhua Xi have made many breakthroughs. They broaden the scope of mathematics disciplines in China beyond traditional strong fields such as geometry and analysis. The helps from many visitors abroad are fundamental to this development. This includes Viehweg, Esnault, Looijenga, and many others.

In geometric analysis, Yum-Tong Siu, Peter Li, Chang-Shou Lin, Kefeng Liu, Xujia Wang, Mu-Tao Wang, Conan Leung, Chiu-Chu Liu, Lizhen Ji, Jixiang Fu, Xiping Zhu, Juncheng Wei and numerous other scholars still perform first-class research. This can clearly be seen from the award winners this year, as well as their speeches. We are grateful to the constant visits of Richard Schoen, Richard Hamilton, Neil Trudinger and others.

In applied mathematics, Zhouping Xin, Sijue Wu, Chongqing Cheng, Chi-Wang Shu, Yizhao Hou, Raymond Chan, and Wen-Wei Lin have contributed excellent work in

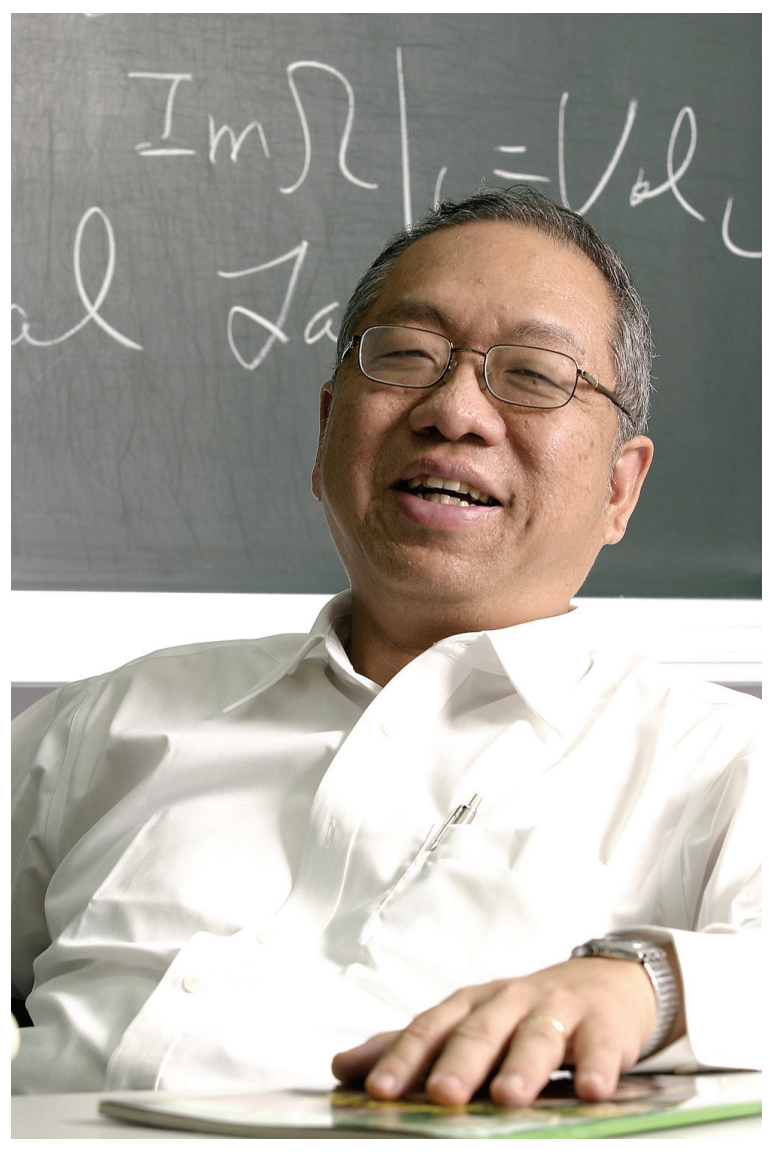

Shing-Tung Yau

(photo by Yiyo Ling, reprinted courtesy of Scientific American, Taiwanese edition)

fluid theory, dynamical systems, computational fluid theory, and numerical linear algebra. In addition to these contributions, Xianfeng $\mathrm{Gu}$ has made outstanding advancements in image processing, as has Stephen Yau and Lei Guo on control theory. Chinese applied mathematicians are no longer just focused on classical finite element methods. The visits of Osher, Engquist, Majda and many 
others have been very useful for our developments.

Under the leadership of Tze-Leung Lai, Wing Hung Wong, Chien-Fu Jeff Wu, Ziliang Ying, Jianqing Fan, and Jun Liu, Chinese statisticians have also attained an important position in the world.

We would like to show our appreciation of the generous support of numerous government leaders and the Morningside Group over the years. We are especially grateful for the strong support of the Chan brothers.

In Mainland China, eighteen years ago, Ronnie Chan, Yongxiang $\mathrm{Lu}$ (the former president of Chinese Academy of Sciences), Lo Yang, and I founded the Morningside Center of Mathematics at the Academy of Sciences in Beijing. There we trained a large class of Chinese scholars, coming from all corners of the country. In fact, the talents leading in the new directions in number theory, which I just mentioned, mostly came to the Morningside Center to learn and to perform research. They were not only from Chinese Academy of Sciences, but also from Peking University, Tsinghua University, Zhongshan University, the University of Science and Technology, Fudan University, Nankai University, Zhejiang University, as well as many other universities. I'm very glad that most of them have gone on to become very good mathematicians, and many of them will deliver speeches in the following five days. After the formation of Morningside Center of Mathematics, several centers of mathematics were formed within the mainland, this includes the center in Zhejiang University, and the Tsinghua Center of Mathematics, where many outstanding mathematicians from Europe and American are joining.

In Taiwan, Professor Zhaoxuan Liu, the former dean of National Science Council of Taiwan, founded the National Center for Theoretical Sciences, at my suggestion. This center is located at the Hsinchu Tsinghua University, and it is split into two divisions: physics and mathematics. The first director was Chang-Shou Lin, who was very successful in fulfilling his duties. Following Chang-Shou Lin, Jing $\mathrm{Yu}$ and Wen-Ching Li became the directors of NCTS, and they both did a tremendous job training many talented young scholars in Taiwan, as well as increasing the rate of communication with overseas scholars. Later on, at National Taiwan University, National Chiao Tung University, and other mathematics research institutes were founded, which also made great contributions to bettering the academic atmosphere.

At the Chinese University of Hong Kong, at my suggestion and with President Charles Kuen Kao's help, the Institute of Mathematics was founded in 1992. Later, it was financed by my fundraising effors through my friends: Robert Kuok Hock Nien, Ka-Shing Li, William Mong Man Wai, Thomas T.T. Chen, and the Hysan Foundation. The Institute hired a group of outstanding mathematicians, and also trained a large number of talented young people. Some of the outstanding students are leading researchers including some Ph.D students who became Benjamin Peirce Fellows at Harvard University.

The first congress was held in Beijing by the Morningside Center of Mathematics, where the leaders of the academy of science and a group of distinguished government officer came to support the activities. Many prominent mathematicians from abroad representing different countries gave speeches.
The second congress was held in Taipei when, at that moment, there were not many Mainland scholars coming to Taiwan due to the problem of visas. In spite of this, all scholars were very enthusiastic about participating in the congress. We celebrated the ninetieth birthday of Professor Shiing-Shen Chern, who was unfortunately not in very good health, and so his daughter $\mathrm{Pu}$ Chen and his son-in-law Paul Ching-Wu Chu attended the congress on his behalf.

The third congress was held in Hong Kong by the Chinese University of Hong Kong. President Yongxiang Lu of the academy of science came to give the opening speech. State councilor Zhi Li Chen sent a letter to congratulate the success of the congress. The congress also decided to memorialize the honorable chairman of the congress, Professor Chern, who had just passed away. Professor Chern has been planning to attend the congress, and had donated one-hundred thousand yuan to the congress.

The fourth congress was held in Hangzhou, and was endorsed by the former party secretaries of the Zhejiang Province, Jinping $\mathrm{Xi}$ and Hongzhu Zhao. Yuanchao Li, minister of the Organization Department of the Central Committee and NPC Vice Chairman Yongxiang Lu both sent congratulatory letters. Governor Zushan Lv and other leaders attended the opening ceremony, as well as the welcoming dinner.

The fifth congress was held in Beijing, and was co-organized by Tsinghua University and the Morningside Center of the Chinese Academy of Sciences. This congress was dedicated to the hundredth anniversaries of the births of Professor Shiing-Shen Chern and of Professor Luogeng Hua. It was held in the Great Hall of the People. There were more than 1,600 participants! Its opening ceremony was truly a grand occasion.

Today the sixth congress is held in Taipei, at a time when mathematical achievements are more prosperous, which is incredibly important. We hope that, when we hold the next congress at the Chinese Academy of Sciences, our native speakers will reach the level of the Fields Medal.

We are pleased to say that fifteen years ago Chang-Shou Lin was the first Chinese mathematician residing in Taiwan to receive the Morningside Gold Award in Beijing. He did most of his work in Taiwan. After waiting fifteen years, we are pleased to see that Ye Tian and $\mathrm{Xu}$ hua He, who reside in Beijing and Hong Kong, respectively, are receiving the Morningside Gold Award in this congress.

It is our honor to see the participation of President Si-Chen Lee, former president of National Taiwan University. He received the Shiing-Shen Chern award, due to his contributions to the mathematical society.

I am sure that many well-to-do Chinese will continue to support this congress, whether through academic works or financially. Chinese mathematics will continue to flourish. I am grateful to the support from all of you. 